\title{
Caracterização epidemiológica da sífilis congênita em Teresina-Piauí no período de
}

\section{3 a 2017}

Epidemiological characterization of congenital syphilis in Teresina-Piauí in the period 2013 to 2017

Caracterización epidemiológica de la sífilis congénita en Teresina-Piauí en el período 2013 a 2017

Recebido: 17/02/2021 | Revisado: 24/02/2021 | Aceito: 28/02/2021 | Publicado: 07/03/2021

Juliana de Morais Araújo

ORCID: https://orcid.org/0000-0003- 4941-8902 Instituto de Consultoria E Educação, Brasil E-mail: julianamorais25@live.com

Aline Correia Matos e Silva ORCID: https://orcid.org/0000-0002- 9498-9061

Centro Universitário UniFacid Wyden, Brasil E-mail: alinecmmatos@hotmail.com

Rosane da Silva Santana

ORCID: https://orcid.org/0000-0002-0601-8223

Universidade Federal do Ceará, Brasil

E-mail: rosane_santana5@hotmail.com

Francisco Alex do nascimento da Silva

ORCID: https://orcid.org/0000-0002-2576-7713

Centro Universitário Maurício de Nassau, Brasil

E-mail: francisco_alex44@hotmail.com

Eliete Carneiro dos Santos

ORCID: https://orcid.org/0000-0002-5384-7614

Centro universitário UNINOVAFAP, Brasil

E-mail: eliete.santos.barros@gmail.com

Ana Cristina Ferreira Pereira

ORCID: https://orcid.org/0000-0002-8429-5496

Instituto de Medicina Integral Fernando Figueira, Brasil

E-mail: annicris874@gmail.com

Adriana de Sousa Brandim

ORCID: https://orcid.org/0000-0003-1486-8903 Fundação Municipal de Saúde, Brasil E-mail: drikabrandim@ hotmail.com

Erica Jorgiana dos Santos de Morais

ORCID: https://orcid.org/0000-0003-2219-7177 Universidade Federal do Piauí, Brasil

E-mail: ericajorgiana@hotmail.com

Angélica Linhares Silva Lima

ORCID: https://orcid.org/0000-0003-2677-8585 Faculdade de Tecnologia e Educação Superior Profissional, Brasil

E-mail: angellinhares09@gmail.com

Maria Oneide dos Santos

ORCID: https://orcid.org/0000-0003-3053-6626

Centro Universitário Maurício Nassau, Brasil

E-mail:neideyy@hotmail.com

Fabíola Pires Lima de Carvalho

ORCID: https://orcid.org/0000-0002-2318-1030

Centro Universitário Maurício de Nassau, Brasil

E-mail: fabiolaespecializacao@gmail.com

Juliene Pereira de Sousa

ORCID: https://orcid.org/0000-0002-0803-1974 Faculdade Aliança, Brasil

E-mail: juliene.p.s@hotmail.com

Patrícia Cristiane Feitosa Rodrigues

ORCID: https://orcid.org/0000-0003-2756-364X

Centro Universitário UniREDENTOR, Brasil

Email: patyenfa2@hotmail.com

\section{Resumo}

A sífilis congênita é uma infecção de múltiplos sistemas causada pelo Treponema pallidum, transmitida ao feto pela placenta, em qualquer período gestacional ou durante o parto. No Brasil, anualmente, observa-se o aumento do 
número de notificações associadas aos casos de sífilis durante a gestação, $80 \%$ com contaminação fetal. Objetivou-se analisar as características epidemiológicas da Sífilis Congênita em Teresina-PI nos anos de 2013 a 2017. Pesquisa descritiva com dados secundários coletados no DATASUS, por meio do Sistema de Informação de Agravos de Notificação (SINAN). Foram incluídas no estudo as variáveis dos RNs/Crianças, da mãe e do parceiro e excluídas somente as variáveis maternas que estavam em análise e não disponibilizadas pelo Sistema. Para a organização dos dados, foi utilizado Tabwin32, tabulador de dados desenvolvido pelo DATASUS/MS. Utilizou-se a estatística descritiva com frequências absolutas, relativas para apresentação dos resultados. Foram encontrados 1.180 casos de SC, sendo que no ano de 2013 apresentou 110 (9,32\%) casos e 2015, 336 (25,82\%). Quanto às características do RNs/crianças, 591 (50,08\%) eram do sexo masculino, $1.133(96,01 \%)$ tinham faixa etária de 0 a seis dias, 755 $(63,98 \%)$ raça parda, $1.003(85 \%)$ residentes na zona urbana, e $1.052(89,15 \%)$ com diagnóstico de SC recente. Verificou-se que 435 (36,86\%) mães tinham baixa escolaridade, $986(83,55 \%)$ realizaram o pré-natal e 800 (67,79\%) parceiros não realizaram o tratamento. Os números de casos elevados demonstram fragilidade na assistência durante o pré-natal com baixa efetividade das ações de promoção e prevenção, bem como tratamento e fatores determinantes no controle da sífilis congênita.

Palavra-chave: Diagnóstico precoce; Gestação; Incidência; Sífilis congênita.; Saúde materno-infantil; Transmissão vertical.

\section{Abstract}

Congenital syphilis is a multiple system infection caused by Treponema pallidum, that it is transmitted to the fetus by the placenta any gestational period or during obstetric labor. In Brazil, there is an increase in the number of reports associated with syphilis during pregnancy and $80 \%$ with fetal contamination. The aim of the study was to analyze the epidemiological characteristics of Congenital Syphilis in Teresina-PI from 2013 to 2017. It is a quantitative research of descriptive nature with secondary data collected in DATASUS, through the Information System for Notification of Injuries (SINAN). The variables of the RNs / Children of the mother and the partner were included in the study, and only the maternal variables that were analyzed and not available by the System were excluded. For the organization of the data, we used Tabwin32, a data tabulator developed by DATASUS / MS. Descriptive statistics were used with absolute, relative frequencies to present the results. There were 1,180 cases of SC, and in 2013, $110(9.32 \%)$ cases and 2015, 336 (25.82\%). The main characteristics identified in the RNs / children were 591 (50.08\%) male cases, 1,133 $(96.01 \%)$ with ages ranging from 0 to 6 days, 755 (63.98\%) brown, 1,003 85\%) living in the urban area, and 1,052 $(89.15 \%)$ with a diagnosis of recent SC. It was verified that $435(36.86 \%)$ mothers had low schooling, $986(83.55 \%)$ had prenatal care and $800(67.79 \%)$ did not receive treatment. The high numbers of cases demonstrate fragility in prenatal care with low effectiveness of promotion and prevention actions, as well as treatment and determinants in the control of congenital syphilis.
\end{abstract}

Keywords: Early diagnosis; Gestation; Incidence; Congenital syphilis; Maternal and child health; Vertical transmission.

\title{
Resumen
}

La sífilis congénita es una infección de múltiples sistemas causada por Treponema pallidum, transmitida al feto por la placenta, en cualquier período gestacional o durante el parto. En Brasil, hay un aumento anual en el número de notificaciones asociadas con casos de sífilis durante el embarazo, 80\% con contaminación fetal. El estudio tuvo como objetivo analizar las características epidemiológicas de la Sífilis Congénita en Teresina-PI en los años 2013 a 2017. Investigación cuantitativa de carácter descriptivo con datos secundarios recolectados en DATASUS, a través del Sistema de Información de Enfermedades Notificables (SINAN). Se incluyeron en el estudio las variables de los RN / Hijos, la madre y la pareja y solo se excluyeron las variables maternas que estaban bajo análisis y no disponibles por el Sistema. Para la organización de los datos se utilizó Tabwin32, una pestaña de datos desarrollada por DATASUS / MS. Se utilizó estadística descriptiva con frecuencias relativas absolutas para presentar los resultados. Se encontraron 1.180 casos de CS, con $110(9,32 \%)$ casos en 2013 y $336(25,82 \%)$ en 2015. En cuanto a las características de los recién nacidos / niños, 591 (50,08\%) eran varones, 1.133 (96,01\%) tenían entre 0 y seis días, 755 (63,98\%) mestizos, $1.003(85 \%)$ residentes en la zona urbana, y $1.052(89,15 \%)$ con diagnóstico reciente de SC. Se encontró que 435 $(36,86 \%)$ madres tenían baja escolaridad, $986(83,55 \%)$ recibieron atención prenatal y $800(67,79 \%)$ parejas no recibieron tratamiento. El elevado número de casos muestra debilidad en la atención durante el prenatal con baja efectividad de las acciones de promoción y prevención, así como el tratamiento y factores determinantes en el control de la sífilis congénita.

Palabras clave: Diagnóstico precoz; Gestación; Incidencia; Sífilis congénita; Salud maternal e infantil; Transmisión vertical. 


\section{Introdução}

No Brasil, o número de notificações associadas aos casos de sífilis durante a gestação aumenta anualmente e $80 \%$ das gestantes apresentam contaminação fetal. No ano de 2016, foram notificados 37.436 casos de sífilis em gestantes e 20.474 de Sífilis Congênita (SC) (Brasil, 2018).

Em decorrência do crescimento da SC, o Ministério da Saúde (MS) lançou o "Plano de ação para eliminação da transmissão vertical do HIV e da Sífilis Congênita”, juntamente com os países da Organização Pan-Americana da Saúde (OPAS) em 2010. O objetivo global do plano de ação era eliminar a SC no ano de 2015, considerando que a incidência de até 0,5 casos a cada mil nascidos vivos eram aceitáveis. No entanto, em 2015 e 2016, respectivamente, foram notificados 6,5 e 6,8 casos a cada mil nascidos vivos, valores distantes da meta proposta (Brasil, 2017).

A Sífilis Congênita é uma infecção de múltiplos sistemas causados pela bactéria espiroqueta gram-negativa e microaerófila conhecida como Treponema pallidum, transmitida ao feto pela placenta, em qualquer período gestacional ou durante o parto (Souza \& Santana, 2013). É de notificação compulsória, responsável por desfechos desfavoráveis e, caso não seja diagnosticada e tratada precocemente, pode causar abortamento, prematuridade, complicações agudas, óbito fetal e outras sequelas para o binômio mãe e filho (Shubert et al., 2018).

O aumento do risco de transmissão e da gravidade da SC ocorre quando a mãe tem sífilis recente, devido ao aumento de espiroquetas na corrente sanguínea. No entanto, nos últimos meses da gestação, a transmissão é menor, devido à imunocompetência de o feto ser maior (Brasil, 2017).

Dentre os fetos contaminados, 20 a 40\% são abortados, 20 a 25\%, natimortos e 15 a 55\% tornam-se Recém-Nascidos (RNs) prematuros. Ressalta-se que aproximadamente $60 \%$ dos RNs com SC são assintomáticos ou sintomáticos ao nascer, o que indica a necessidade de valorização da anamnese materna e interpretação adequada do teste sorológico realizado durante o pré-natal. Entre a $3^{\mathrm{a}}$ e $8^{\mathrm{a}}$ semana, $60 \%$ dos RNs podem desenvolver sintomas e $40 \%$ poderão desenvolver SC precoce ou tardia (Rodriguez-Cerdeira \& Silami-Lopes, 2012).

As manifestações da SC precoce iniciam-se até os dois anos de idade na criança exposta. Nos primeiros dias de vida, o RN pode apresentar os casos mais graves como prematuridade, icterícia, anemia, lesão cutaneomucosas, hepatoesplenomegalia, sofrimento respiratório com ou sem pneumonia relacionada à resposta inflamatória à infecção ativa do agente (Brasil, 2018; Domingues, Saraceni, HartzIII \& Carmo LealIV, 2013).

A SC tardia refere-se ao quadro iniciado após os dois anos de vida, correspondendo à sífilis terciária, resultante da cicatrização do processo inflamatório e/ou hipersensibilidade. Geralmente, nessa fase, a resposta ao tratamento é insatisfatória, assemelhando-se com as manifestações presentes nos adultos, exceto pelo raro acometimento cardiovascular (RodriguezCerdeira \& Silami-Lopes, 2012).

Para o diagnóstico da sífilis congênita, são associados critérios epidemiológicos, clínicos, laboratoriais e devem ser avaliados a história clínica-epidemiológica da mãe, o exame físico dos RNs/ crianças e os resultados dos exames laboratoriais e radiológicos (Lima, Santos, Barbosa \& Ribeiro, 2013).

Existem duas categorias de testes, os não treponêmicos, sendo o principal Veneral Deseases Research Laboratory (VDRL), que corresponde aos testes de floculação fundamentados na identificação de anticorpos contra a cardiolipina, considerados quantitativos, sensíveis, baratos e necessários para o acompanhamento da terapêutica; e os testes treponêmicos, que são qualitativos e detectam anticorpos contra os treponemas, conhecidos como confirmatório, podendo ser solicitados uma vez durante a doença (Pedrosa, 2010).

O Ministério da Saúde preconiza que os testes não treponêmicos sejam disponibilizados na primeira consulta do prénatal até o primeiro trimestre de gestação, os outros, no início do terceiro trimestre e na sala de parto (Brasil, 2015a). Nos 
recém-nascidos com suspeita de sífilis congênita, a avaliação diagnóstica deve ser baseada nos exames VDRL, que é realizado pela coleta sanguínea periférica, radiografias de ossos longos, análise de líquor cefalorraquidiano e hemograma (Feitosa, Rocha \& Costa, 2016).

Após a confirmação do diagnóstico da sífilis na gestante, o tratamento é realizado com penicilina benzatina, que tem a capacidade de atravessar a barreira transplacentária, tratando mãe e feto ao mesmo tempo, com dose padrão de 2.400 UI distribuídos nos dois glúteos, por via intramuscular (IM), e também uma droga de escolha para RNs/crianças que tem caso confirmado. Quando as gestantes e os RNs/crianças apresentarem alergia à medicação penicilina benzatina, a ceftriaxona $1.000 \mathrm{mg}$ é a alternativa (Cavalcante, Pereira \& Castro, 2017).

O Ministério da Saúde preconiza que sejam realizadas consultas de pré-natal com exames minuciosos para todos recém-nascidos com mães soropositivas para sífilis, e que os mesmos possam ser acompanhados durante dois anos no mínimo, com disponibilização de testes sorológicos não treponêmicos (Quin et al., 2014; Brasil, 2017).

A principal ação para o controle da doença é a assistência de pré-natal abrangente e de qualidade, assegurando o diagnóstico precoce e o tratamento adequado em tempo oportuno, com medidas relativamente simples e eficazes na prevenção e tratamento (Brasil, 2018).

A pesquisa teve como objetivo analisar as características epidemiológicas da Sífilis Congênita no município de Teresina-PI nos anos de 2013 a 2017.

\section{Metodologia}

Trata-se de uma pesquisa quantitativa de natureza descritiva. O estudo descritivo facilita a descrição de fatos e fenômenos da realidade estudada, por meio de pesquisa tem como ênfase a elucidação de significados, que permitem a consideração de aspectos diferentes de uma situação ou problema (Gil, 2017).

$\mathrm{O}$ enfoque quantitativo de pesquisa representa um conjunto de processos sequencial e comprobatório. Cada etapa antecede à seguinte e não pode ultrapassar os passos, a sequência é rigorosa, porém, pode redefinir alguma fase. Usa-se a coleta de dados para avaliar hipóteses, fundamentando na medição numérica e na análise estatística para determinar padrões e comprovar teorias (Sampieri, Collado \& Lucio, 2013).

A pesquisa foi realizada com dados secundários sobre sífilis congênita no munícipio de Teresina-PI, coletados no Departamento de Informação do Sistema Único de Saúde (DATASUS), por meio do Sistema de Informação de Agravos de Notificação (SINAN). Foram incluídas no estudo as variáveis dos RNs/Crianças, da mãe e do parceiro entre os anos de 2013 a 2017 e excluídas somente as variáveis maternas que estavam em análise e não disponibilizadas pelo Sistema. A coleta de dados aconteceu nos meses de dezembro de 2018 a março de 2019, pelas pesquisadoras, utilizando-se um formulário fechado elaborado exclusivamente para fins da pesquisa (APENDICE A).

Foram empregadas as seguintes variáveis dos RNs/crianças: sexo, zona, raça, faixa etária e classificação final; maternas: escolaridade, realização do pré-natal, momento do diagnóstico; e paternas, apenas a realização do tratamento. Em todas as variáveis foi utilizada a subvariável ignorada, correspondendo aos dados que não foram preenchidos, porém não comprova que esses dados foram ignorados pelos profissionais ou os próprios sujeitos.

Para a organização dos dados quantitativos, foi utilizado o programa Tabwin32 do Departamento de Informática do Sistema Único de Saúde (DATASUS), que tabula dados de diferentes tipos como de internação hospitalar, mortalidade, população e etc., e por conseguinte o programa Microsoft Excel, versão XP (Microsoft CO, USA). Os dados coletados foram importados para o Programa SPSS "Statistical Package for the Social Science" (versão 20.0 for Windows), software que possibilita calcular o percentual dos dados encontrados e realizar a análise estatística. 
Foi utilizada a estatística descritiva com frequências absolutas e relativas. Após análise, os dados foram organizados e apresentados em forma de gráficos e tabelas, com objetivo de facilitar a interpretação.

O estudo foi realizado por meio de dados secundários do banco de dados de domínio público do SINAN-DATASUS, o que assegura a preservação da identidade dos sujeitos e princípios éticos, estabelecidos pela Resolução $466 / 2012$ do Conselho Nacional de Saúde, que define as diretrizes e normas regulamentadoras de pesquisas, incluindo preferencialmente seres humanos (Brasil, 2012). Assim não houve necessidade avaliação do Comitê de Ética em Pesquisa (CEP).

\section{Resultados e Discussão}

De acordo com os dados registrados no SINAN-DATASUS/MS, entre os anos de 2013 a 2017 foram encontrados 1.180 casos de sífilis congênita em Teresina-PI.

Gráfico 1 - Número de casos de Sífilis Congênita nos anos de 2013 a 2017 (n=1.180), Teresina-PI.

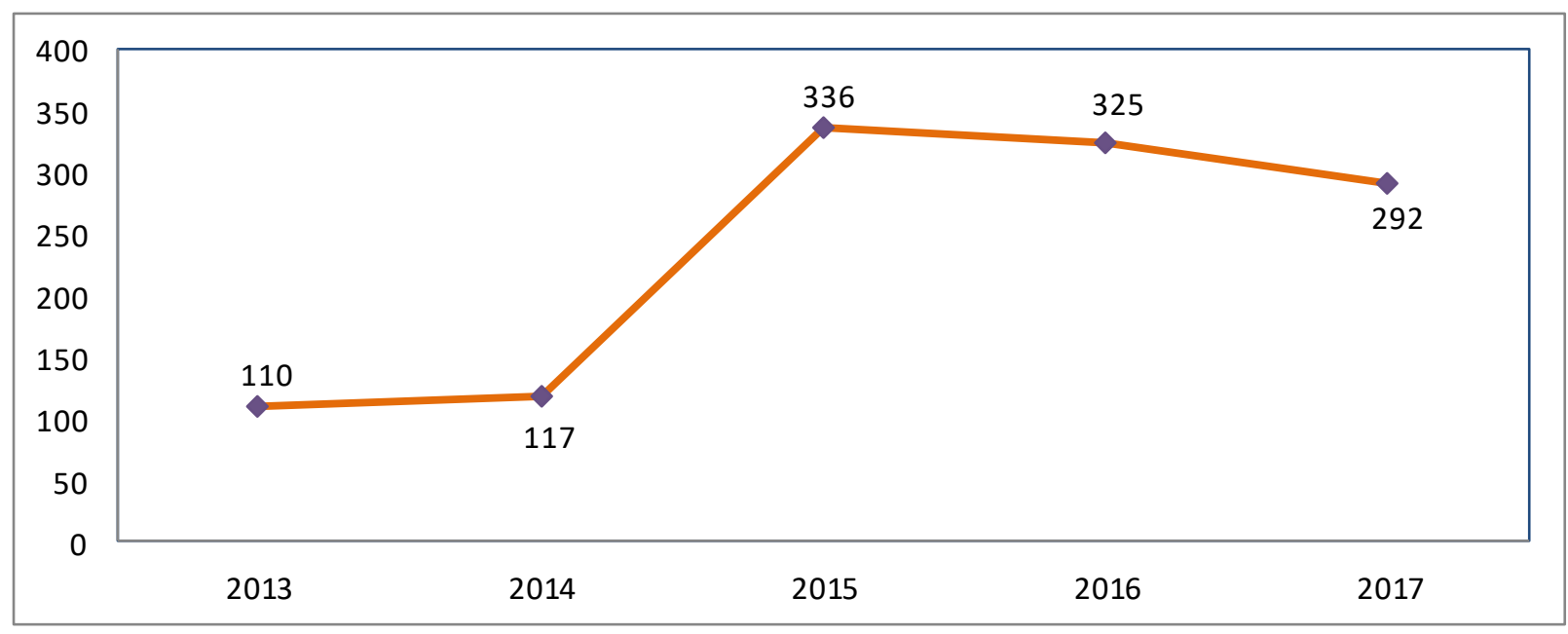

Fonte: DATASUS/SINAN.

No Gráfico 1, observou-se que o número de casos de SC no ano de 2015 foi maior, com 336 (25,82\%) e no ano de 2013 menor, 110 (9,32\%). Nos anos de 2016 e 2017 houve uma queda no número de casos comparados a 2015.

Na pesquisa de Cardoso et al., (2018), em Fortaleza-CE, foram encontrados 1528 casos de sífilis congênita entre os anos de 2008 a 2010. Resultados semelhantes foram verificados no estudo de Alves et al., (2016) no estado de Alagoas, com 1.258 casos de SC entre os anos de 2007 a 2011.

Almeida et al., (2015) relataram que a ocorrência de casos de sífilis congênita associa-se ao manejo inadequado da gestante, com perda da oportunidade de diagnosticar e tratar em tempo oportuno na Atenção Básica, evidenciando-se que precisa haver melhorias nas políticas de atenção básica e educação em saúde para minimizar a incidência da doença.

Os dados do estudo mostraram que os casos de sífilis congênita até o ano de 2015 foram crescentes, quando comparados a estudos similares, não correspondendo com as metas da Organização Pan-Americana de Saúde (OPAS) para a erradicação da doença. O ministério da saúde determinou objetivos para a redução dos números de casos de sífilis congênita, como cobertura de pré-natal, pelo menos uma consulta, cobertura para testagem rápida e cobertura de tratamento com penicilina em gestantes com sífilis, com pretensão de atingir um valor igual ou >95\% (Brasil, 2015b).

Em virtude das metas propostas pela OPAS, no ano de 2011 foi instituída a rede cegonha com objetivo de garantir à mãe e à criança cuidados integrados, garantindo uma atenção humanizada durante o acompanhamento do pré-natal, do parto, no nascimento, puerpério e atenção à criança no âmbito do sistema de saúde. Um de seus planos era assegurar a implantação de 
testes rápidos para a triagem sorológica de sífilis durante o pré-natal, garantindo um diagnóstico em tempo oportuno e prevenção da transmissão vertical da sífilis congênita (Melo \& Santos, 2015).

$\mathrm{O}$ crescimento da SC pode estar relacionado à falta de assistência ou procura tardia para realização do pré-natal, não tratamento concomitante do parceiro, tratamentos inadequados, falta de capacitação de profissionais tanto para diagnosticar, quanto para notificar os casos.

Tabela 1: Descrição dos dados sociodemográficos dos recém-nascidos e das crianças com Sífilis Congênita entre os anos de 2013 e 2017. $(n=1.180)$, Teresina-PI.

\begin{tabular}{lcccccccccccc}
\hline Variáveis & \multicolumn{2}{c}{$\mathbf{2 0 1 3}$} & \multicolumn{2}{c}{$\mathbf{2 0 1 4}$} & \multicolumn{2}{c}{$\mathbf{2 0 1 5}$} & \multicolumn{2}{c}{$\mathbf{2 0 1 6}$} & \multicolumn{2}{c}{$\mathbf{2 0 1 7}$} & \multicolumn{2}{c}{ Total } \\
\hline Faixa etária & & & & & & & & & & & & \\
Até 6 dias & 99 & 8,3 & 114 & 9,6 & 327 & 27,7 & 313 & 26,5 & 280 & 23,72 & 1.133 & 96 \\
7-27dias & 2 & 0,1 & 1 & 0,08 & 2 & 0,2 & 5 & 0,4 & 6 & 0,5 & 16 & 1,3 \\
28 dias <1ano & 9 & 0,7 & 2 & 0,2 & 7 & 0,6 & 7 & 0,6 & 6 & 0,5 & 31 & 2,6 \\
Sexo & & & & & & & & & & & & \\
Masculino & 59 & 5 & 51 & 4,3 & 185 & 15,6 & 163 & 13,8 & 133 & 11,2 & 591 & 50 \\
Feminino & 44 & 3,7 & 57 & 4,8 & 139 & 11,7 & 144 & 12,2 & 147 & 12,4 & 531 & 45 \\
Ignorado & 7 & 0,6 & 9 & 0,7 & 12 & 1,0 & 18 & 1,5 & 12 & 1,0 & 58 & 4,9 \\
Zona & & & & & & & & & & & & \\
Urbana & 95 & 8,0 & 105 & 8,9 & 281 & 23,8 & 272 & 23,0 & 250 & 21,1 & 1.003 & 85 \\
Rural & 14 & 1,1 & 5 & 0,4 & 52 & 4,4 & 50 & 4,2 & 34 & 2,8 & 155 & 13,1 \\
Periurbana & 0 & 0 & 0 & 0 & 1 & 0,08 & 0 & 0 & 0 & 0 & 1 & 3,08 \\
Ignorado & 1 & 0,1 & 7 & 0,6 & 2 & 0,2 & 3 & 0,2 & 8 & 0,6 & 21 & 1,7 \\
Raça & & & & & & & & & & & & \\
Branca & 23 & 1,9 & 32 & 2,7 & 44 & 3,7 & 75 & 6,3 & 35 & 2,9 & 209 & 17,7 \\
Preta & 8 & 0,7 & 9 & 0,7 & 16 & 1,3 & 5 & 0,4 & 11 & 0,9 & 49 & 4,1 \\
Amarela & 0 & 0 & 1 & 0,08 & 3 & 0,2 & 0 & 0 & 0 & 0 & 4 & 0,3 \\
Parda & 66 & 5,6 & 60 & 5,0 & 236 & 20 & 202 & 17,1 & 191 & 16,1 & 755 & 64 \\
Indígena & 0 & 0 & 0 & 0 & 2 & 0,2 & 0 & 0 & 0 & 0 & 2 & 0,2 \\
Ignorado & 13 & 1,1 & 14 & 1,2 & 35 & 2,9 & 43 & 3,6 & 55 & 4,6 & 161 & 13,6 \\
\hline
\end{tabular}

Fonte: DATASUS/SINAN.

Na Tabela 01, a faixa etária com maior percentual de sífilis congênita foi em neonato, com seis dias de nascido no total de $1.133(96,0 \%)$. Houve predominância do sexo masculino, com 591 (50\%) casos; e raça, cor parda, com 755 (64\%) casos, seguida da cor branca, com 209 (17,7\%) casos avaliados. Quanto à zona de habitação, a urbana apresentou maior relevância, com $1.003(85 \%)$ e a periurbana, com um $(0,08 \%)$ caso. 
Silva, Jesus Teodoro, Silva, Silva Santos e Olinda (2017), em um estudo realizado no Mato Grosso do Sul, mostraram que $51 \%$ das crianças com SC apresentavam-se com 0 dias de vida, sendo que a maioria era do sexo feminino 78 (51\%) e raça parda, $84(54,9 \%)$ casos.

Referente à raça, Rodrigues, Lima, Costa e Batista (2014) encontraram na população do município de São Luiz-MA 286 crianças nascidas com SC, com predominância da parda, com 78,8\% dos casos, corroborando o estudo de Silva et al., (2017) e Rocha, Terças, Nascimento, Silva e Gleriano (2016), que identificaram no município de Tangará da Serra-MT que 63,6\% eram do sexo masculino, apresentando dados semelhantes do estudo.

Concernente à residência, nota-se que há um número de casos de SC na zona urbana (85\%). O dado encontrado assemelhou-se com o de Cardoso, Araújo, Cavalcante, Frota e Melo (2018) em estudo realizado no estado do Ceará, com predominância dos casos confirmados residentes na zona urbana (74,68\%), e ao do estudo de Teixeira (2016), realizado com 39 casos de SC no município de Jequié-BA, entre os anos de 2007 a 2013, observou-se que 69\% estavam inseridos na zona urbana do território investigado.

Verificou-se que o número de casos na zona urbana foi bastante elevado, considerando os recursos amplificados que são destinados à população. Porém as periferias dos grandes centros urbanos são locais propícios para o acometimento de Infecções Sexualmente Transmissíveis, possivelmente, resultante da dificuldade de acesso à informação. Por conseguinte, pôde-se identificar os locais com maior número de casos e a partir disso investir numa educação em saúde a fim de promover a redução desse agravo.

Ressalta-se que a sífilis não é uma infecção seletiva, ou seja, qualquer sexo está vulnerável a adquirir a SC, pois o que determina a infecção transplacentária é o tratamento da mãe. Portanto, é importante a efetuação do pré-natal com qualidade e tratamento adequado, que oriente a gestante e o parceiro sobre a infecção e os riscos aos quais o feto está exposto independente do sexo, raça, faixa etária e zona dos RNs/crianças.

Tabela 2: Percentual da classificação final da sífilis congênita em Recém-nascidos e crianças entre os anos de 2013 e 2017. $(\mathrm{n}=1.180)$, Teresina-PI.

\begin{tabular}{|c|c|c|c|c|c|c|c|c|c|c|c|c|}
\hline \multirow[b]{2}{*}{ Variável } & \multicolumn{2}{|c|}{2013} & \multicolumn{2}{|c|}{2014} & \multicolumn{2}{|c|}{2015} & \multicolumn{2}{|c|}{2016} & \multicolumn{2}{|c|}{2017} & \multicolumn{2}{|c|}{ Total } \\
\hline & $\mathrm{N}$ & $\%$ & $\mathrm{n}$ & $\%$ & n & $\%$ & $\mathrm{n}$ & $\%$ & $\mathrm{n}$ & $\%$ & $\mathrm{n}$ & $\%$ \\
\hline SC recente & 82 & 6,9 & 103 & 8,7 & 296 & 25 & 295 & 25 & 276 & 23,3 & 1.052 & 89,1 \\
\hline $\begin{array}{l}\text { Natimorto/ } \\
\text { Aborto }\end{array}$ & 8 & 0,6 & 6 & 0,5 & 11 & 0,9 & 15 & 1,2 & 8 & 0,6 & 48 & 4,0 \\
\hline Ignorado & 20 & 1,7 & 8 & 0,6 & 29 & 2,4 & 15 & 1,2 & 8 & 0,6 & 80 & 6,7 \\
\hline
\end{tabular}

Fonte: DATASUS/SINAN.

A Tabela 2 mostrou que os casos de SC recente correspondem a $1.052(89,1 \%)$ e foi notificado natimorto/aborto por sífilis apenas 48 (4,0\%). Amâncio, Graciano, Cozer, Assis e Silva Dias (2016) constataram, no município de Goiás, 481 (89\%) casos de sífilis congênita recente, cinco (1\%) casos de natimorto/aborto. Em Tangará da Serra-MT, Rocha et al., (2016) encontraram 11 casos, sendo que nenhum apresentou sintomas da doença e nem houve natimorto/aborto por sífilis.

O quadro clínico varia e depende do tempo de exposição fetal ao Treponema, ou seja, duração da sífilis na gestação sem tratamento, carga treponêmica materna e virulência do treponema. A maior parte das crianças nascidas com sífilis pode ser assintomática ao nascer e apresentar sinais muito discretos ou pouco específicos (França et al., 2015). 
No entanto, é necessário ressaltar que os sinais e sintomas podem se manifestar de forma precoce e tardia após os dois anos de idade (Brasil, 2015b). Como foi apresentado em vários estudos que a maior parte dos casos de SC são assintomáticos ao nascer, no estudo durante o período de anos selecionados, 89,15\% dos casos apresentaram SC recente, que se tratados adequadamente, apresentarão eficácia e poucos gastos financeiros, se comparados aos consumos utilizados nas unidades de terapia intensiva dos recém-nascidos acometidos por esta infecção.

Verifica-se a necessidade da realização periódica do monitoramento dos RNs/crianças, com consultas clínicas bimensais; realização de testes não treponêmicos até os 18 meses de idade; monitoramento das funções neurológicas, oftalmológicas e auditivas, conforme preconizado pelo MS; e que o profissional de saúde esteja atento a todas as possíveis manifestações clínicas, adotando-se medidas adequadas para reduzir sequelas à criança.

Tabela 3: Descrição do nível de escolaridade materna dos casos de Sífilis Congênita entre os anos de 2013 e 2017. (n=1.180). Teresina-PI.

\begin{tabular}{|c|c|c|c|c|c|c|c|c|c|c|c|c|}
\hline \multirow{2}{*}{ Variável } & \multicolumn{2}{|c|}{2013} & \multicolumn{2}{|c|}{2014} & \multicolumn{2}{|c|}{2015} & \multicolumn{2}{|c|}{2016} & \multicolumn{2}{|c|}{2017} & \multicolumn{2}{|c|}{ Total } \\
\hline & $\mathbf{n}$ & $\%$ & $\mathbf{n}$ & $\%$ & $\mathbf{n}$ & $\%$ & $\mathbf{n}$ & $\%$ & $\mathbf{n}$ & $\%$ & $\mathbf{n}$ & $\%$ \\
\hline \multicolumn{13}{|l|}{ Escolaridade } \\
\hline Analfabeto & 0 & 0 & 1 & 0,0 & 3 & 0,2 & 1 & 0,08 & 2 & 0,1 & 7 & 0,6 \\
\hline $\begin{array}{l}\text { Ens. Fundamental } \\
\text { Incompleto }\end{array}$ & 42 & 3,5 & 37 & 3,1 & 129 & 11 & 142 & 12,0 & 85 & 7,2 & 435 & 36,8 \\
\hline $\begin{array}{l}\text { Ens. Fundamental } \\
\text { Completo }\end{array}$ & 20 & 1,7 & 23 & 1,9 & 55 & 4,6 & 33 & 2,7 & 50 & 4,2 & 181 & 15,3 \\
\hline $\begin{array}{l}\text { Ensino Médio } \\
\text { Incompleto }\end{array}$ & 19 & 1,6 & 21 & 1,7 & 73 & 6,1 & 59 & 5 & 56 & 4,7 & 228 & 19,3 \\
\hline $\begin{array}{l}\text { Ensino Médio } \\
\text { Completo }\end{array}$ & 15 & 1,2 & 17 & 1,4 & 51 & 4,3 & 50 & 4,2 & 46 & 3,9 & 179 & 15,1 \\
\hline $\begin{array}{l}\text { Educação Superior } \\
\text { Incompleta }\end{array}$ & 1 & 0,08 & 1 & 0,08 & 7 & 0,6 & 7 & 0,6 & 4 & 0,3 & 20 & 1,6 \\
\hline $\begin{array}{l}\text { Educação Superior } \\
\text { Completa }\end{array}$ & 1 & 0,08 & 0 & 0 & 2 & 0,2 & 3 & 0,2 & 2 & 0,2 & 8 & 0,7 \\
\hline Ignorado & 12 & 1,0 & 17 & 1,4 & 16 & 1,3 & 30 & 2,5 & 47 & 3,9 & 122 & 10,3 \\
\hline
\end{tabular}

Fonte: DATASUS/SINAN.

A Tabela 3 mostrou que 435 (36,8\%) mães tinham o nível de escolaridade até o ensino fundamental incompleto e 228 $(19,3 \%)$ estudaram até o ensino médio incompleto. Os dados assemelharam-se aos de uma pesquisa realizada entre 2010 e 2013 em Belo Horizonte-MG, resultados de outros estudos que confirmaram a maior ocorrência de casos de SC em mães com baixa escolaridade (Nonato, Melo \& Guimarães, 2014; Gonzales, Tapia \& Serruya, 2015).

Serafim et al., (2014) ressaltaram que a baixa escolaridade é considerada um indicador de maior risco para disseminação das infecções sexualmente transmissíveis, devido a um limitado entendimento da importância das medidas de prevenção.

No estado da Bahia, nos anos de 2010 a 2013, Oliveira e Santos (2015) verificaram que 367 mães, dos 1.594 casos, apresentaram baixo nível de instrução até 8 anos. Porém, a grande maioria dos registros refletiu a falta de capacitação dos 
profissionais quanto ao conhecimento ao domínio das fichas, como também da indispensabilidade e importância da notificação desses dados, devido a apresentação como ignorados. Esses métodos inadequados atrapalham o controle da SC, resultando, basicamente, no desconhecimento da doença e seu tratamento pelas gestantes.

Domingues et al., (2013) relataram que a dificuldade de abordagem das infecções sexualmente transmissíveis no período gestacional e, talvez, o desconhecimento das gestantes quanto aos danos que podem prejudicar a saúde, no binômio mãe e bebê, constituem assim outras dificuldades para o controle da SC. Destaca-se que, na assistência à gestante com sífilis, os aspectos como o comportamento sociocultural, que vai além dos recursos humanos, material e médico, precisam ser considerados.

A sífilis congênita tem sido relacionada ao baixo nível de escolaridade materna, consequentemente, quanto maior o grau de escolaridade menor o índice da doença, como descrito nos dados coletados no estudo. Apesar dessa doença não ser restrita às classes sociais menos favorecidas, os resultados do estudo sinalizam que podem ser marcadores importantes de pouco acesso ao serviço da saúde, mesmo aqueles que vivem em zona urbana como citado anteriormente. Considerando o índice elevado de baixa escolaridade, é incontestável que as mães possuem pouco conhecimento relacionado às medidas de promoção, prevenção, tratamento e reabilitação das infecções sexualmente transmissíveis.

Tabela 4: Número de casos de sífilis materna, segundo realização do pré-natal, período do diagnóstico e realização do tratamento do parceiro entre os anos de 2013 e 2017. ( $\mathrm{n}=1.180)$, Teresina-PI.

\begin{tabular}{lcccccccccccc}
\hline Variáveis & $\mathbf{2 0 1 3}$ & \multicolumn{2}{c}{$\mathbf{2 0 1 4}$} & \multicolumn{2}{c}{$\mathbf{2 0 1 5}$} & \multicolumn{2}{c}{$\mathbf{2 0 1 6}$} & \multicolumn{2}{c}{$\mathbf{2 0 1 7}$} & \multicolumn{2}{c}{ Total } \\
\hline Realizou Pré-natal & & & & & & & & & & & & \\
Sim & 88 & 7,5 & 92 & 7,8 & 274 & 23,2 & 278 & 23,5 & 254 & 21,5 & 986 & 83,5 \\
Não & 19 & 1,6 & 25 & 2,1 & 61 & 5,1 & 46 & 3,9 & 36 & 3,0 & 187 & 15,8 \\
Ignorado & 3 & 0,2 & 0 & 0 & 1 & 0,08 & 1 & 0,08 & 2 & 0,2 & 7 & 0,6 \\
Tratamento & & & & & & & & & & & & \\
Parceiro & & & & & & & & & & & & \\
Sim & 23 & 1,9 & 32 & 2,7 & 67 & 5,6 & 86 & 7,3 & 96 & 8,1 & 304 & 25,7 \\
Não & 74 & 6,3 & 80 & 6,7 & 261 & 22,1 & 218 & 18,5 & 167 & 14,1 & 800 & 67,8 \\
Ignorado & 13 & 1,1 & 5 & 0,4 & 8 & 0,6 & 21 & 1,8 & 29 & 2,4 & 76 & 6,4 \\
Momento do & & & & & & & & & & & & \\
Diagnóstico & & & & & & & & & & & & \\
Pré-natal & 44 & 3,7 & 43 & 3,6 & 144 & 12,2 & 157 & 13,3 & 141 & 11,9 & 529 & 44,8 \\
Parto & 36 & 3,0 & 49 & 4,1 & 147 & 12,4 & 139 & 11,7 & 115 & 9,7 & 486 & 41,1 \\
Após o parto & 25 & 2,1 & 22 & 1,8 & 40 & 3,4 & 26 & 2,2 & 30 & 2,5 & 143 & 12,1 \\
Não realizado & 2 & 0,2 & 0 & 0 & 4 & 0,3 & 1 & 0,08 & 3 & 0,2 & 10 & 0,8 \\
Ignorado & 3 & 0,2 & 3 & 0,2 & 1 & 0,08 & 2 & 0,16 & 3 & 0,2 & 12 & 1,0 \\
\hline
\end{tabular}

Fonte: DATASUS/SINAN.

Tabela 4 mostrou que $986(83,5 \%)$ mães efetuaram o pré-natal, 800 (67,8\%) parceiros não receberam tratamento e 529 (44,8\%) mães receberam o diagnóstico no pré-natal. Segundo Barbosa, Almeida, Silva, Araújo e Santos (2017), grande 
parte dos casos de SC onde às mães tinham efetuado o pré-natal, não garantiu o tratamento das mesmas e de seus parceiros. Lafetá, Martelli Júnior, Silveira e Paranaíba (2016) ressaltaram também que maioria das gestantes realizaram o pré-natal, porém, 38\% delas foram diagnosticadas com sífilis no momento do parto.

Almeida et al., (2015) demonstraram os mesmos resultados, com a realização do pré-natal conforme preconizado, mas, devido alguns fatores que dificultam o diagnóstico durante o pré-natal, os quais podem ser relacionados ao baixo número de consultas ,ausência do exame VDRL no primeiro e terceiro trimestre gestacional, atraso dos laboratórios na entrega dos resultados ,ausência de retorno da gestante com os resultados dos exames, bem como a falha do serviço na busca das gestantes que abandonam o acompanhamento do pré-natal e ainda a falta de estrutura nas unidades de tratamento.

Almeida et al., (2015) também identificaram maior predominância de casos de sífilis congênita relacionados ao não tratamento do parceiro, correspondendo a 58,90\%, após ser diagnosticado com a doença. Concordando com o estudo de Lafetá et al., (2016), que apresentou 31(33,3\%) parceiros os quais não realizaram o tratamento, e 62 mulheres foram consideradas inadequadamente tratadas, pois $98 \%$ dos seus parceiros não realizaram o tratamento.

Outras investigações realizadas por Lima et al., (2017) e Silva et al., (2017) evidenciaram o mesmo perfil, com elevado número de parceiros que não receberam tratamento adequado para sífilis, e relataram evidências que não há participação efetiva dos mesmos nos serviços de saúde durante o "pré-natal" ou, em alguns casos, o anonimato/não ciência do parceiro pela gestante. Carvalho (2016) relatou que a participação do parceiro durante o "pré-natal" deveria ser uma estratégia prioritária dos profissionais que o realizam, tendo em vista a diminuição do risco de reinfecção da gestante durante o tratamento. Esse fator predominante impede que as taxas de transmissão vertical sejam reduzidas, tornando o controle da SC limitado, tendo em vista o grande número de não tratados.

Como verificado no estudo, a predominância dos casos de SC aconteceu com mães que realizaram o pré-natal, mas não houve garantia de cura e muito menos impossibilitou a transmissão vertical. Observou-se por meio dos dados uma falha nos serviços de acompanhamento do pré-natal e de vários fatores citados anteriormente, apresentando dificuldade de manejar, diagnosticar e tratar oportunamente as gestantes e seus parceiros.

É notável a necessidade do reforço à inclusão do parceiro sexual no pré-natal, como estratégia para melhoria da cobertura de testagem e tratamento adequado de ambos e consequente redução da transmissão vertical, principalmente após alteração da definição de caso pelo MS, que passou a incluir recentemente a testagem do parceiro. Evidenciando falha no sistema durante a escuta qualificada, educação em saúde, aconselhamento de seus usuários e vigilância em saúde. Todos esses fatores reunidos contribuem para a disseminação da sífilis e por conseguinte da sífilis congênita.

\section{Conclusão}

Com base no número elevado de casos de Sífilis Congênita em Teresina-PI, verifica-se que há fragilidade na assistência durante o pré-natal, com baixa efetividade das ações de prevenção e tratamento relacionados aos fatores determinantes no controle dessa infecção.

As políticas de implementação devem ser desenvolvidas na melhoria do diagnóstico precoce da sífilis em gestante, com consequente diminuição no número de Sífilis congênita, nos custos com tratamento, na capacitação da equipe multidisciplinar de saúde na rede da Atenção Básica, criação de protocolos de saúde atualizados que possam promover e implementar atividades educativas individuais e coletivas para captação precoce de gestantes, adoção de práticas de prevenção, adesão das gestantes e parceiros aos tratamentos de doenças transmissíveis e no pré-natal. Alerta-se também para a subnotificação ou não alimentação do Sistema, havendo necessidade de maior vigilância eficaz para evitá-los. 


\section{Referências}

Almeida, P. D., Araujo Filho, A. C. A., Araújo, A. K. L., Carvalho, M. L., Silva, M. G. P., \& Araújo, T. M. E. (2015). Epidemiological analysis of congenital syphilis in Piauí. Revista Interdisciplinar, 8(1), 62-70.

Alves, W. A., Cavalcanti, G. R., Andrade Nunes, F., Teodoro, W. R., Carvalho, L. M., \& Domingos, R. S. (2016). Sífilis Congênita: Epidemiologia dos Casos Notificados em Alagoas, Brasil, 2007 a 2011. Revista Portal: Saúde e Sociedade, 1(1), 27-41.

Amâncio, V. C., Graciano, A. R., Cozer, A. M., Assis, L. P. F., \& Silva Dias, D. C. (2016). Epidemiology of congenital syphilis in the state of goiás. Revista Educação em Saúde, 4(2).

Barbosa, D. R. M., Almeida, M. G. D., Silva, A. O., Araújo, A. A., \& Santos, A. G. D. (2017). Epidemiological profile of cases of gestational syphilis. Rev. enferm. UFPE on line, 1867-1874.

Cardoso, A. R. P., Araújo, M. A. L., Cavalcante, M. D. S., Frota, M. A., \& Melo, S. P. D. (2018). Analysis of cases of gestational and congenital syphilis between 2008 and 2010 in Fortaleza, State of Ceará, Brazil. Ciência \& Saúde Coletiva, 23, 563-574.

Carvalho, A. M. P. (2016). Representações sociais de trabalhadores da Estratégia de Saúde da Família sobre o princípio da equidade no cuidado em saúde aos portadores de doenças sexualmente transmissíveis (Dissertação de mestrado, Universidade Federal de Juiz de Fora, Juiz de Fora, MG, Brasil).

Cavalcante, P. A. D. M., Pereira, R. B. D. L., \& Castro, J. G. D. (2017). Syphilis in pregnancy and congenital syphilis in Palmas, Tocantins State, Brazil, 2007-2014. Epidemiologia e Serviços de Saúde, 26, 255-264.

Domingues, R. M. S. M., Saraceni, V., HartzIII, Z. M. A., \& Carmo LealIV, M. Sífilis congênita: evento sentinela da qualidade da assistência pré-natal. Ver Saúde Pública, 47(1),147-157.

França, I. S. X. D., Batista, J., Coura, A. S., Oliveira, C. F. D., Araújo, A. K. F., \& Sousa, F. S. D. (2015). Fatores associados à notificação da sífilis congênita: um indicador de qualidade da assistência pré-natal, Rev Rene,16(3),374-81.

Gil, A. C. (2002). Como elaborar projetos de pesquisa. Atlas.

Gonzales, G. F., Tapia, V., \& Serruya, S. J. (2014). Sífilis gestacional y factores asociados en hospitales públicos del Perú en el periodo 2000-2010. Revista Peruana de Medicina experimental y salud pública, 31, 211-221.

Hernández Sampieri, R., Fernández Collado, C., \& Baptista Lucio, P. (2013). Metodologia de pesquisa. Tradução por Daisy Vaz de Moraes, 5.

Lafetá, K. R. G., Martelli Júnior, H., Silveira, M. F., \& Paranaíba, L. M. R. (2016). Sífilis materna e congênita, subnotificação e difícil controle. Revista Brasileira de Epidemiologia, 19, 63-74.

Lima, M. G., Santos, R. F. R. D., Barbosa, G. J. A., \& Ribeiro, G. D. S. (2013). Incidência e fatores de risco para sífilis congênita em Belo Horizonte, Minas Gerais, 2001-2008. Ciência \& Saúde Coletiva, 18, 499-506.

Lima, V. C., Mororó, R. M., Martins, M. A., Ribeiro, S. M., \& Linhares, M. S. C. (2017). Perfil epidemiológico dos casos de sífilis congênita em um município de médio porte no nordeste brasileiro. Journal of Health \& Biological Sciences, 5(1), 56-61.

Mello, V. S. D., \& Santos, R. D. S. (2015). A sífilis congênita no olhar da enfermagem. Rev. enferm. UERJ, 23(5), 699-704.

Ministério da Saúde, Brasil. (2012). Conselho Nacional de Saúde. Resolução n 466, de 12 de dezembro de 2012 . Aprova normas regulamentadoras de pesquisas envolvendo seres humanos: Diário Oficial da União.

Ministério da Saúde, Brasil. (2015a) Secretaria de Ciência, Tecnologia e Insumos Estratégicos. Protocolo Clínico e Diretrizes Terapêuticas Infecções Sexualmente Transmissíveis: Ministério da Saúde.

Ministério da Saúde, Brasil. (2015b). Boletim Epidemiológico SÍfILIS. Brasília.

Ministério da Saúde, Brasil. (2017). Boletim Epidemiológico SÍFILIS 2017. Brasília.

Ministério da Saúde, Brasil. (2018) Secretaria de Vigilância em Saúde. Departamento de DST, Aids e Hepatites Virais. Protocolo Clinico e Diretrizes Terapêuticas para Atenção Integral as Pessoas com Infecções Sexualmente Transmissíveis. Brasília.

Nonato, S. M., Melo, A. P. S., \& Guimarães, M. D. C. (2015). Sífilis na gestação e fatores associados à sífilis congênita em Belo Horizonte-MG, 20102013. Epidemiologia e Serviços de Saúde, 24 (4), 681-694.

Oliveira, J. S., \& Santos, J. V. (2015). Perfil Epidemiológico Da Sífilis Congênita No Estado Da Bahia, No Período De 2010a 2013. Rev. Eletrôn. Atualiza Saúde, 2(2).

Pedrosa, L. D. C. O. (2010). Sífilis congênita: fatores de risco em gestantes admitidas nas maternidades de Maceió/AL e área Metropolitana e avaliação dos critérios diagnósticos adotados no Brasil. 139f. Tese (Doutorado em Medicina Tropical). Universidade Federal de Pernambuco. Recife.

Qin, J. B., Feng, T. J., Yang, T. B., Hong, F. C., Lan, L. N., Zhang, C. L., \& Dong, W. (2014). Risk factors for congenital syphilis and adverse pregnancy outcomes in offspring of women with syphilis in Shenzhen, China: a prospective nested case-control study. Sexually transmitted diseases, 41(1), 13-23.

Rocha, R. P. S., Terças, A. C. P., Nascimento, V. D., Silva, J. D., \& Gleriano, J. S. (2007). Análise do perfil epidemiológico de sífilis nas gestantes e crianças. Tangará da Serra, de, 03-21. 
Research, Society and Development, v. 10, n. 3, e9710313068, 2021

(CC BY 4.0) | ISSN 2525-3409 | DOI: http://dx.doi.org/10.33448/rsd-v10i3.13068

Rodrigues, L. D. S., Lima, R. H. D. S., Costa, L. C., \& Batista, R. F. L. (2014). Características das crianças nascidas com malformações congênitas no município de São Luís, Maranhão, 2002-2011. Epidemiologia e Serviços de Saúde, 23(2), 295-304.

Rodríguez-Cerdeira, C., \& Silami-Lopes, V. G. (2012). Congenital syphilis in the 21st century. Actas Dermo-Sifiliográficas (English Edition), 103(8), 679693.

Serafim, A. S., Moretti, G. P., Serafim, G. S., Niero, C. V., Rosa, M. I. D., Pires, M. M. D. S., \& Simões, P. W. T. D. A. (2014). Incidence of congenital syphilis in the South Region of Brazil. Rev da Soc Bras Med Trop, 47(2), 170-178.

Shubert, C. O., Silva, S. L., Carvalho, C. M. S. M., Cunha, J. C., Santos, L. F. D. M., \& Silva, C. B. F. (2018). Transmissão vertical da sífilis: o enfermeiro e as ações de prevenção. Rev Científica Multidisciplinar do Centro Universitário São José, 11(1).

Silva, J. A.F., Rocha, C. H. R., \& Costa, F. S. (2016). Artigo de revisão: Sífilis congênita. Rev Med Saúde de Brasília, 5(2), $286-97$.

Silva, L. C. V. G., Jesus Teodoro, C. C., Silva, J. K., Silva Santos, D. A., \& Olinda, R. A. (2017). Profile of the cases of syphilis in a municipality the South of Mato Grosso. Journal Health NPEPS, 2(2), 380-390.

Souza, B. C., \& Santana, L. S. (2013). As consequências da sífilis congênita no binômio materno-fetal: um estudo de revisão. Interfaces Científicas-Saúde e Ambiente, 1(3), 59-67.

Teixeira, M. A., Santos, P. P., Santos, P. N., Araújo, R. T., \& Souz, A. G. J. (2015). Perfil epidemiológico e sociodemográfico das crianças infectadas por sífilis congênita no município de Jequié/Bahia. Rev Saúde Com, 303-13. 